\title{
The validation of the Hungarian version of the ID-migraine questionnaire
}

\author{
Éva Csépány ${ }^{1,3}$, Marianna Tóth², Tamás Gyüre ${ }^{1}$, Máté Magyarr ${ }^{1,3}$, György Bozsik $^{3}$, Dániel Bereczki ${ }^{3}$, \\ Gabriella Juhász ${ }^{4,5}$ and Csaba Ertsey ${ }^{3^{*}}$
}

\begin{abstract}
Background: Despite its high prevalence, migraine remains underdiagnosed and undertreated. ID-Migraine is a short, self-administrated questionnaire, originally developed in English by Lipton et al. and later validated in several languages. Our goal was to validate the Hungarian version of the ID-Migraine Questionnaire.

Methods: Patients visiting two headache specialty services were enrolled. Diagnoses were made by headache specialists according to the ICHD-3beta diagnostic criteria. There were 309 clinically diagnosed migraineurs among the 380 patients. Among the 309 migraineurs, 190 patients had only migraine, and 119 patients had other headache beside migraine, namely: 111 patients had tension type headache, 3 patients had cluster headache, 4 patients had medication overuse headache and one patient had headache associated with sexual activity also. Among the 380 patients, 257 had only a single type headache whereas 123 patients had multiple types of headache. Test-retest reliability of the ID-Migraine Questionnaire was studied in 40 patients.
\end{abstract}

Results: The validity features of the Hungarian version of the ID-Migraine questionnaire were the following: sensitivity 0.95 (95\% Cl, 0.92-0.97), specificity 0.42 (95\% Cl, 0.31-0.55), positive predictive value 0.88 (95\% Cl, 0.84-0.91), negative predictive value 0.65 (95\% Cl, 0.5-0.78), missclassification error 0.15 (95\% Cl, 0.12-0.19). The kappa coefficient of the questionnaire was 0.77 .

Conclusion: The Hungarian version of the ID-Migraine Questionnaire had adequate sensitivity, positive predictive value and misclassification error, but a low specificity and somewhat low negative predictive value.

Keywords: ID-migraine questionnaire, Hungarian version, Validity features, Migraine

\section{Background}

Migraine is a common disease, which affects $14 \%$ of the population, and $18 \%$ of women [1] globally. In the USA, its lifetime prevalence is $25 \%$ in women [2]. It affects mainly the active, working, young adult population [3]. In Hungary, only one population based headache epidemiology study has been made to date [4]. This study reported $67 \%$ lifetime prevalence for any kind of headache: the one-year prevalence of migraine without aura was $7.6 \%$, and the one-year prevalence of migraine with aura was $2 \%$. Only $43 \%$ of migraineurs had ever consulted any physician because of their headache, and 15\%

\footnotetext{
* Correspondence: csaba.ertsey@gmail.com

${ }^{3}$ Department of Neurology, Semmelweis University, Balassa u. 6, Budapest 1083, Hungary
}

Full list of author information is available at the end of the article of patients missed school or work because of their headache in the previous year.

According to the report of Global Burden of Disease studies, migraine is the third cause of disability in 1549 years old men and women [5]. The disability, caused by migraine, affects many aspects of life, and leads to both physcial and emotional impairment [6]. In one Swedish study, researchers found that in migrainous patients, the health-releated quality of life is significantly worse, not only during the migraine attacks, but also between attacks, compared to healthy controls. In another study, $65 \%$ of migraine patients reported some degree of absenteeism from their workplace or school due to their headache [6]. The indirect costs of migraine are considerable: in the USA alone, approximately 13 billion dollars are spent each year for migrainre-related absenteeism from workplace and reduced ability to work 
[7]. Despite migraine's serious negative effects on the individual, less than half of the patients ever recieve a medical diagnosis of headache $[8,9]$. Furthermore, migraine is suboptimally treated, with only one-third receiving some kind of migraine-specific medication [10]. There are several factors of migraine being underdiagnosed and undertreated. The most important factor is that many migraine patients - even those with quite strong headaches - do not consult their doctors because of their headache, and therefore do not receive the diagnosis of migraine [8]. In the UK, $4.4 \%$ of the population would see a general practitioner because of a headache problem in a given year, $34 \%$ of whom (ie. $1.5 \%$ of the population) would be prescribed a migraine medication (acute or prophylactic), while only $2.1 \%$ of those who consult would be referred to a neurologist [11]. This compares to a $14.3 \%$ one-year prevalence of migraine in the UK [12]: the reason for the low consultation rates is not self-evident [11]. The severity of the attack may not be a decisive factor in consulting: an American survey found that $61 \%$ of those who had never consulted reported severe or very severe pain and 67\% reported severe disability or the need for bed rest during their migraines [13]. A further difficulty in diagnosing migraine may be the duration of the doctor-patient meeting, which may not be enough to discuss the characteristics of the headaches. Furthermore, a number of primary care physicians may not have an adequate knowledge about headaches [14], and the IHS criteria are excessively complex and time-consuming for routine application in primary care $[15,16]$.

In order to facilitate the detection of migraine in primary care, Lipton et al. (2003) developed a brief, selfadministered questionnaire, the ID-Migraine [17]. The questionnaire contains two pre-screening questions, one asking about headache-related disability, the other asking whether the patient would like to consult a doctor because of the headache. This is followed by three screening questions pertaining to the previous three months. These screening questions ask about headache-related disability, nausea and sensitivity of light. The ID-Migraine indicates migraine if a patient answered "yes" at least to two of the three screening questions. In the original study, the sensitivity of the ID-Migraine Questionnaire was 0.81, the specificity was 0.75 , and the positive predictive value was 0.93 . Test-retest reliability was good, with a kappa of 0.68 . The ID-Migraine Questionnaire was therefore found to be a valid, reliable, and easy-to-use screening instrument to detect migraine, for patients presenting in primary care. The authors emphasized that the ID-Migraine Questionnaire is not a diagnostic instrument by itself, so a thorough evaluation of patients is necessary to make the diagnosis of migraine. Subsequently, the questionnaire was validated in Italian [18], Portuguese [19] and Turkish [20] languages with adequate results (Table 1). The questionnaire has been used in many specialty fields to screen migraine, not only in primary care $[16,21]$, but also in headache centers and neurology clinics [18, 19, 22]. Moreover, the questionnaire was successfully used for screening migraine patients in the emergency department [23], in a temporomandubular and orofacial pain clinic [24], and also in opthalamic and ear, nose and throat clinics [25]. The ID-Migraine Questionnaire proved to be reliable not only in adults, but also among adolescents [26]. It was also used in large-scale studies of migraine epidemiology [21] and genetic studies [27].

In this study we present the validity features of the Hungarian version of the ID-Migraine Questionnaire.

\section{Methods}

Patients between 18 and 65 years of age, presenting at the Headache Service of the Department of Neurology, Semmelweis University or the Headache Service of Esztergom Hospital, and reporting two or more headaches in the previous 3 months were involved. Both Services worked with the same methology. In order to include at least 300 patients in the study (ie. 100 patients per questionnaire item, which is a widely accepted and conservative way of assuring an adequate sample size in validation studies [28] we involved all patients visiting these Services in a two-year period who were willing to participate and gave informed consent to processing their results. The study protocol had been approved by the ethics committee of Semmelweis University. Patients completed the questionnaire at the occasion of their medical visit. Most of the patients filled in the questionnaire before the medical visit, ie. while they were waiting to be seen by the neurologist, who, on the other hand,

Table 1 Validation results of the ID-Migraine questionnaire in different languages

\begin{tabular}{llllll}
\hline & Location of the research & Sensitivity $(95 \% \mathrm{Cl})$ & Specificity $(95 \% \mathrm{Cl})$ & $\mathrm{PPV}(95 \% \mathrm{Cl})$ & $\mathrm{NPV}(95 \% \mathrm{Cl})$ \\
\hline English,2003[17] & primary care & $0.81(0.77$ to 0.85$)$ & $0.75(0.64$ to 0.84$)$ & $0.93(0.90$ to 0.96$)$ & $\mathrm{NI}$ \\
Italian, 2007[18] & headache centers & $0.95(0.91$ to 0.98$)$ & $0.72(0.62$ to 0.82$)$ & $0.88(0.82$ to 0.93$)$ & $0.87(0.78$ to 0.95$)$ \\
Turkish, 2007[19] & neurology outpatient clinics & $0.92(\mathrm{NI})$ & $0.63(\mathrm{NI})$ & $0.72(\mathrm{NI})$ & $0.88(\mathrm{NI})$ \\
Portuguese, 2008[20] & headache outpatient clinics & $0.94(0.87$ to 0.97$)$ & $0.60(0.46$ to 0.73$)$ & $0.80(0.71$ to 0.87$)$ & $0.85(0.70$ to 0.94$)$ \\
\hline
\end{tabular}

$\mathrm{Nl}=$ no information, $P P V=$ positive predictive value, $N P V=$ negative predictive value, $\mathrm{Cl}$ confidence interval

Note: NPV was not reported in the English study, classification errors were not reported in any of the studies, and no confidence intervals were reported in the Turkish study 
did not use the questionnaire to ascertain the diagnosis that was based on the interview with the patient. A minority of the patients filled in the questionnaire while they were waiting for their written documentation, ie. after the medical visit. The patients also completed a 9-item Hungarian migraine screener (the MDX questionnaire), developed and validated by our group [29], which was used to collect information about the clinical characteristics of their headaches in more detail, but was not included as a reference tool in the validation process of ID-Migraine. All the patients underwent detailed internal medicine and neurological examination. The gold standard was the neurologists' clinical diagnosis, according to the International Classification of Headache Disorders (ICHD3- $\beta$ ). As in the original English version [17], the Hungarian version of the ID-Migraine was considered positive for migraine if a patient answered "yes" at least to two of the three screening questions. The responses to the ID-Migraine Questionnaire were then compared with the clinical diagnosis of migraine: if a patient had a diagnosis of migraine, she/he was considered a migraineur regardless of having other headaches beside migraine or not. Based on the primary diagnosis, the questionnaire's sensitivity, specificity, positive predictive value (PPV), negative predictive value (NPV), and classification error were calculated. These parameters were calculated for the individual items of the ID-Migraine Questionnaire, as well. Based on previously reported validation studies $[18,19]$ we also calculated these values in subgroups of patients according to sex, age (equal or below 44 years and above 44 years) and disease duration (equal or below 12 years and above 12 years) in order to have a more thorough vision of the Hungarian version's performance.

In addition, to evaluate the characteristics of the Hungarian ID-Migraine Questionnaire the receiver operating curve (ROC) was constructed among the 380 patients with different sensitivity (true positive rate) and 100 -specificity (false positive rate) values according to the minimum number of positive answers to the ID-Migraine Questionnaire (0, 1, 2, and 3 positive answers).

Among the 380 headache sufferers, 40 patients completed the ID-Migraine Questionnaire twice, the second time was also during a follow-up visit. Test-retest reliability, using the Cohen's Kappa, was calculated in these 40 patients. The following values of Cohen's Kappa were used to evaluate the level of agreement [30]: <0: no agreement; $0.0-0.20$ : slight agreement; $0.21-0.40$ : fair agreement; $0.41-0.60$ : moderate agreement; $0.61-0.80$ : substantial agreement; 0.81-1.0: perfect agreement.

We used an Excel spreadsheet for data input, and an online statistical package (VassarStats, http://vassarstats.net/) to calculate the ID-Migraine's validity features (sensitivity, specificity, PPV, NPV), the confidence intervals thereof, misclassification error and test-retest reliability.

\section{Results}

A total of 380 patients completed the Hungarian version of the ID-Migraine Questionnaire. Among the 380 patients, $80 \%$ were female and $20 \%$ were male. The median age was 36 years, the interquartile range was 19.8 years. The median disease duration was 10 years, the interquartile range was 16 years.

Table 2 summarizes the clinical headache diagnoses among the 380 patients. The number of clinically diagnosed migraineurs was 309; among them, 190 had only migraine, whereas 119 patients had another headache diagnoses beside migraine. The total number of non-migraine patients was 71 ; the primary diagnosis was tension type headache (TTH) in 45 patients, cluster headache in 19 patients, and other headache in $7 \mathrm{pa}$ tients. Among the 380 patients, 257 had only one type headache, namely: 190 patients had only migraine, 44

Table 2 Clinical headache diagnoses among the 380 patients who completed the ID-Migraine questionnaire

\begin{tabular}{|c|c|c|c|}
\hline Primary diagnosis & Number & Secondary diagnosis & Number \\
\hline \multirow[t]{5}{*}{ Migraine } & \multirow[t]{5}{*}{309 (251 episodic and 58 chronic) } & none & 190 \\
\hline & & tension type headache & 111 \\
\hline & & cluster headache & 3 \\
\hline & & medication overuse headache & 4 \\
\hline & & headache associated with sexual activity & 1 \\
\hline \multirow[t]{2}{*}{ Tension type headache } & \multirow[t]{2}{*}{45 (12 episodic and 33 chronic) } & none & 44 \\
\hline & & headache associated with sexual activity & 1 \\
\hline \multirow[t]{3}{*}{ Cluster headache } & \multirow[t]{3}{*}{19 (18 episodic and 1 chronic) } & none & 16 \\
\hline & & tension type headache & 2 \\
\hline & & SUNCT syndrome & 1 \\
\hline Other headache & 7 & none & 7 \\
\hline
\end{tabular}


Table 3 The number of ID-Migraine Questionnaire positive patients in clinically diagnosed headache groups

\begin{tabular}{ll}
\hline Clinically diagnosed patients & $\begin{array}{l}\text { ID-Migraine Questionnaire } \\
\text { positive patients }\end{array}$ \\
\hline total sample size: $n=380$ & $n=334$ \\
migraine group: $n=309$ & $n=293$ \\
tension type group: $n=45$ & $n=23$ \\
cluster type group: $n=19$ & $n=16$ \\
other headache group: $n=7$ & $n=2$ \\
\hline
\end{tabular}

had only TTH, 16 had only cluster headache and 7 had only other type of headache. The other 123 patients had more than one type of headache at the time of the study.

Among the 380 patients, 334 had a positive ID-Migraine score; 293 of them had a clinical diagnosis of migraine. Among the 45 patients, clinically diagnosed with TTH, 23 had a positive ID-Migraine score, as did 16 of the 19 patients, whose clinical diagnosis was cluster headache. Table 3 contains the number of positive ID-Migraine Questionnaires in the clinical headache groups.

Figure 1 shows the ROC curve with different cut off points $(0,1,2$, or 3 "yes" answere) to demonstrate the characteristics of the Hungarian ID-Migraine Questionnaire. To calculate validity measures we used the original cutoff value of at least two "yes" answers out of the three screening questions as reported by Lipton et al. [17].
Based on the whole sample $(n=380)$, the quality scores of the Hungarian version of the ID-Migraine Questionnaire were the following: sensitivity 0.95 (95\% CI, $0.92-$ 0.97 ), specificity 0.42 ( $95 \% \mathrm{CI}, 0.31-0.55)$, positive predictive value (PPV) 0.88 (95\%CI, 0.84-0.91), negative predictive value (NPV) 0.65 (95\% CI, 0.5-0.78), misclassification error 0.15 (95\%CI, 0.12-0.19).

Fourty of the 380 patients also completed the questionnaire during a follow-up visit. In this sample, the clinical diagnoses were as follows: 31 patients had migraine, 6 had TTH, 2 had cluster headache, and one had other (cervicogenic) headache. Two of the patients' clinical migraine diagnoses changed between the first and second ID-Migraine Questionnaire assessments: one had migraine as the initial diagnosis and TTH at follow-up, the other had TTH as the initial diagnosis and migraine at follow-up. The median interval between filling out the ID-Migraine Questionnaire for the first and second time was 90.5 days, the interquartile range was 475 days. At the time of the first completion, 36 of the 40 patients had a positive ID-Migaine Questionnaire. At the second time, 34 of the 40 patients had a positive test. The kappa coefficient of the questionnaire was 0.77 , indicating a substantial agreement between the assesments. The overall percent of agreement was 0.95 , the percent of positive agreement was 0.94 .

Table 4 summarizes the quality scores for the separate items of the ID-Migraine Questionnaire using the data

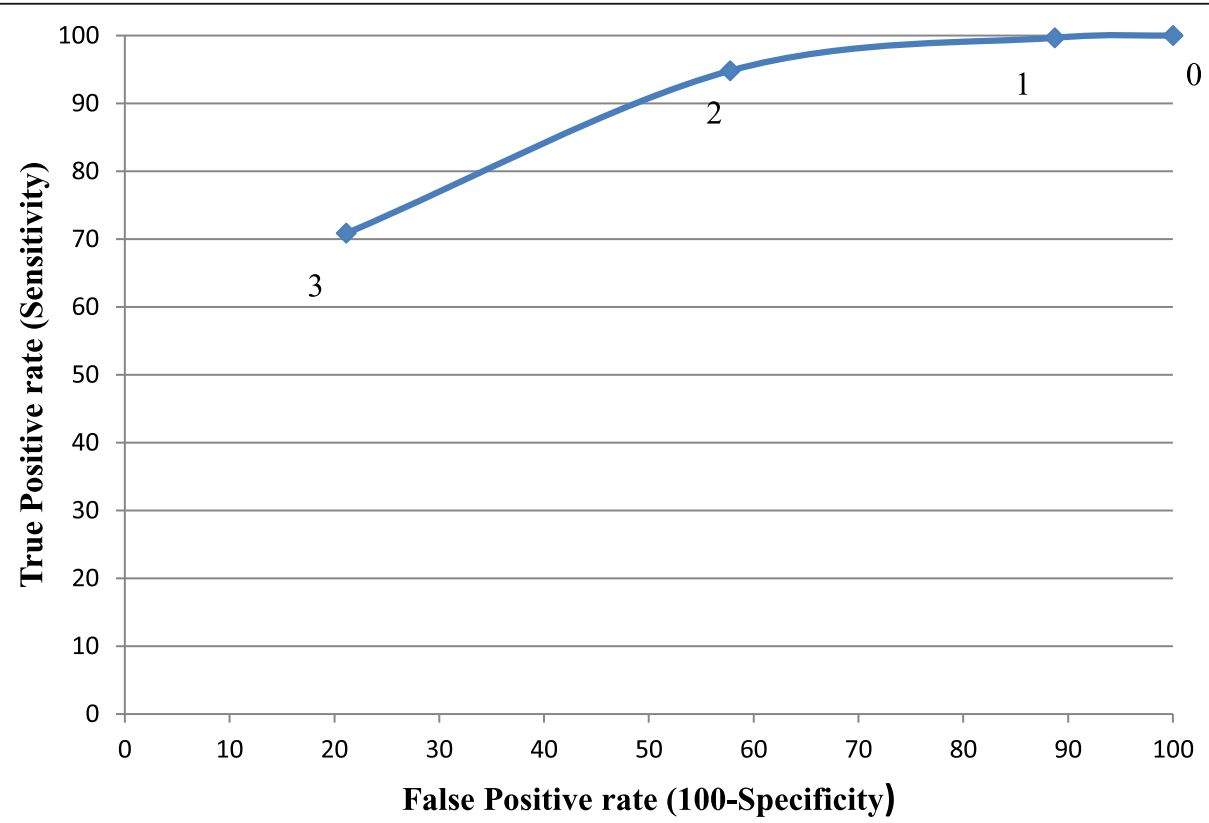

Fig. 1 The receiver operating curve of the ID-Migraine Questionnaire in the study population. The curve shows the sensitivity and specificity values according to the minimum number of positive answers for the ID-Migraine Questionnaire. Thresholds were determined according to the minimum number of positive answers to the ID-Migraine Questionnaire (0,1, 2 and 3 positive answers). 0: sensitivity: 1.00 (95\% Cl; 0.98-1.00); specificity: 0.00 (95\% Cl; 0.00-0.06). 1: sensitivity: 0.997 ( $95 \%$ Cl; 0.98-1.00); specificity: 0.11 (95\% Cl; 0.05-0.22). 2: sensitivity: 0.95 (95\% Cl; 0.92-0.97); specificity: 0.42 ( $95 \%$ Cl; $0.31-0.55$ ). 3: sensitivity: 0.71 ( $95 \%$ Cl; $0.65-0.76$ ); specificity: 0.79 ( $95 \%$ Cl; 0.67-0.87) 
Table 4 Quality scores separately for the items of the Hungarian version of the ID-Migraine Questionnaire $(n=380)$

\begin{tabular}{llllll}
\hline & Sensitivity $(95 \% \mathrm{Cl})$ & Specificity $(95 \% \mathrm{Cl})$ & $\mathrm{PPV}(95 \% \mathrm{Cl})$ & NPV $(95 \% \mathrm{Cl})$ & Classification error (95\% Cl) \\
\hline Nausea & $0.86(0.82-0.90)$ & $0.59(0.47-0.70)$ & $0.9(0.86-0.93)$ & $0.49(0.38-0.60)$ & $0.19(0.15-0.23)$ \\
Photophobia & $0.83(0.78-0.86)$ & $0.58(0.45-0.69)$ & $0.89(0.85-0.93)$ & $0.43(0.33-0.54)$ & $0.22(0.18-0.27)$ \\
Disability & $0.97(0.94-0.98)$ & $0.15(0.08-0.26)$ & $0.83(0.79-0.87)$ & $0.52(0.3-0.74)$ & $0.18(0.15-0.23)$ \\
$\begin{array}{l}\text { ID-migraine positive } \\
(\geq 2 \text { "yes") }\end{array}$ & $0.95(0.92-0.97)$ & $0.42(0.31-0.55)$ & $0.88(0.84-0.91)$ & $0.65(0.5-0.78)$ & $0.15(0.12-0.19)$
\end{tabular}

$(\geq 2$ "yes")

$P P V=$ positive predictive value, $N P V=$ negative predictive value, $\mathrm{Cl}$ confidence interval

of 380 patients. All of the items (nausea, photophobia and disability) had high sensitivity and positive predictive value (>0.8). We found the highest sensitivity for the disability (0.97), similarly to the Italian ID-Migraine Questionnaire. Nausea showed the highest positive predictive value (0.9). By contrast, we found signifcantly lower scores for the negative predictive value and specificity compared to sensitivity and positive predictive value.

Table 5 presents the quality scores of the questionnaire in the clinically relevant subgroups, namely according to sex, age (equal or below 44 years and above 44 years) and disease duration (equal or below 12 years and above 12 years). While the sensitivity and specificity of the ID-Migraine was the same in female and male patients, the PPV was noticeably higher, whereas the NPV and misclassification error were noticeably lower in females than males. There were no other substantial differences between the subgroups.

Given that the Hungarian version of the ID-Migraine had substantially lower specificity and NPV than the original and previous translations, we scrutinized those patients whose were diagnosed with nonmigraine headaches, especially whose ID-Migraine scores were positive, as was the case of $51 \%$ of TTH patients, $84 \%$ of cluster headache patients, and $29 \%$ of other headache patients, making use of the MDX questionnaire that contained the clinical characteristics of their headaches in more detail. Table 6 shows the clinical characteristics of these patients' headaches. All patients in all diagnostic groups reported an at least moderate severity of headache, regardless of their ID-Migraine score. In the TTH group, patients with a positive ID-Migraine score had an average of 3.3 migrainous features, compared to 2.0 in their ID-Migraine negative peers. In the cluster headache group, patients with a positive ID-Migraine score had, on average, 4.0 migrainous features, compared to 0.3 in ID-Migraine negative patients. In the group of other non-migraine headaches, patients with a positive ID-Migraine score had an average of 3.5 migrainous features, whereas the ID-Migraine negative patients had 2.0.

In the TTH group, 23 patients (4 episodic and 19 chronic) had a positive ID-Migraine score. Based on the clinical characteristics available from the MDX questionnaires, 14 patients ( 1 episodic and 13 chronic) could be considered as having migraines as well, whereas 3 episodic and 6 chronic TTH patients did not meet the criteria of migraine. The difference between the distribution of suspected migraine and no migraine was not significant (Chi square test: $p=0.106$ ).

In the cluster headache group, 16 patients (15 episodic and 1 chronic) had a positive ID-Migraine score. As all of these patients were followed up at the Dept. of Neurology, and their clinical documentation was available, we performed a retrospective chart review to ascertain the diagnosis. Based on the clinical characteristics (strictly unilateral attacks; presence of ipsilateral autonomic features; periodicity) the diagnosis of cluster headace was confirmed in all of them. However, as $84 \%$ of cluster

Table 5 Quality scores of the Hungarian version of the ID-Migraine questionnaire in the clinically relevant subgroups

\begin{tabular}{|c|c|c|c|c|c|c|}
\hline & N (\%) & Sensitivity $(95 \% \mathrm{Cl})$ & Specificity (95\% Cl) & PPV (95\% Cl) & NPV (95\% Cl) & Classification error ( $95 \% \mathrm{Cl})$ \\
\hline \multicolumn{7}{|l|}{ Sex } \\
\hline Women & $304(80 \%)$ & $0.95(0.92-0.97)$ & $0.47(0.30-0.65)$ & $0.93(0.90-0.96)$ & $0.55(0.36-0.73)$ & $0.1(0.07-0.14)$ \\
\hline Men & $76(20 \%)$ & $0.95(0.81-0.99)$ & $0.39(0.24-0.56)$ & $0.63(0.49-0.75)$ & $0.88(0.60-0.98)$ & $0.32(0.22-0.44)$ \\
\hline \multicolumn{7}{|l|}{ Age } \\
\hline$\leq 44$ years & 262 (69\%) & $0.96(0.92-0.98)$ & $0.44(0.29-0.60)$ & $0.89(0.84-0.93)$ & $0.69(0.48-0.85)$ & $0.13(0.09-0.18)$ \\
\hline$>44$ years & $118(31 \%)$ & $0.94(0.86-0.98)$ & $0.48(0.27-0.69)$ & $0.87(0.78-0.93)$ & $0.69(0.41-0.88)$ & $0.16(0.10-0.24)$ \\
\hline \multicolumn{7}{|c|}{ Duration of illness } \\
\hline$\leq 12$ years & $228(60 \%)$ & $0.96(0.89-0.99)$ & $0.41(0.25-0.59)$ & $0.82(0.74-0.89)$ & $0.78(0.52-0.93)$ & $0.18(0.12-0.26)$ \\
\hline$>12$ years & $152(40 \%)$ & $0.94(0.85-0.98)$ & $0.33(0.09-0.69)$ & $0.93(0.84-0.97)$ & $0.38(0.10-0.74)$ & $0.13(0.06-0.21)$ \\
\hline
\end{tabular}


Table 6 The self-reported headache characteristics of the non-migraine patients versus their ID-Migraine status

\begin{tabular}{llllllll}
\hline Clinical diagnosis & ID-Migraine & Number of patients & Worse with movement & Nausea & Vomiting & Photophobia & Phonophobia \\
\hline Tension type headache & positive & 23 & 14 & 17 & 1 & 12 & 9 \\
& negative & 22 & 12 & 1 & 0 & 2 & 12 \\
\multirow{3}{*}{ Cluster headache } & positive & 16 & 9 & 9 & 8 & 0 & 1 \\
\multirow{5}{*}{ Other headache } & negative & 3 & 0 & 0 & 0 & 1 & 2 \\
& positive & 2 & 1 & 0 & 0 & 0 & 3
\end{tabular}

Note: All patients reported an at least moderate severity of pain so this was not included In Table 6

headache patients had a positive ID-Migraine score and they were overrepresented in the sample, we also calculated the quality scores excluding these patients. In this calculation, sensitivity was 0.95 (95\%CI: 0.92-0.97), specificity was 0.52 (95\% CI: $0.38-0.66)$, PPV was 0.92 (95\% CI: $0.88-0.95)$, NPV was 0.63 (95\% CI: $0.47-0.77)$ and misclassification error was 0.11 (95\%CI: 0.08-0.15).

Finally the two patients who had other non-migrainous headaches and a positive ID-Migraine score could be considered as having migraines as well, based on the characteristics of their headaches.

\section{Discussion}

Our results demonstrated that the Hungarian version of the ID-Migraine Questionnaire is a reliable screening instrument for migraine based on data collected at specialist headache centres. The fact that all patients fully completed the questionnaire indicates that it is easy to understand and use, so it could be used as a screening tool in primary care, and also for research purposes. The sensitivity and positive predictive value of the Hungarian version of the ID-Migraine Questionnaire were quite similar to those of the original English version [17], with the Hungarian version having a higher sensitivity (0.95 vs. 0.81$)$. The classification error (which had not been reported in the previous validation studies) was also acceptable. On the other hand, the specificity of the Hungarian version was markedly lower, and the negative predictive value was also somewhat lower than in the previous validation studies. It is important to note, that our sample was quite similar to other validation studies where the participants had also been recruited from headache centers [18-20, 29, 31-34].

All of the items (nausea, photophobia and disability) had high sensitivity and positive predictive value $(>0.8)$. This is in agreement with the Italian and Portugese versions of ID-Migraine Questionnaire [18, 19]. We found the highest sensitivity for the disability (0.97), similarly to the Italian ID-Migraine Questionnaire [18]. The nausea showed the highest positive predictive value (0.9), similarly to the portugese version of ID-Migraine Questionnaire [19], which supports the previous studies that headache-related nausea is an important accompaining symptom and has high impact on migraine [8, 35-39].

From the 309 clinically diagnosed migraine patients, 293 had positive ID-Migraine scores. This result supports the previous findings, that ID-Migraine Questionnaire has a high screening accuracy [17-19, 22, 25, 40-42].

In addition, the quality scores of the questionnaire showed no significant difference between clinically relevant subgroups, divided by sex, age and disease duration, similarly to the Portuguese and Italian versions of ID-Migraine Questionnaire [18, 19]. This suggests that the questionnaire may be used in the general population.

The cause of the lower specificity and NPV of the Hungarian version ( 0.42 vs 0.75 for the English version) may in part be due to the high number of false positive patients among the clinically diagnosed cluster headache and TTH patients. In particular, cluster headache was overrepresented in the sample (the prevalence of cluster headache patients in the sample was $5.0 \%$ versus a roughly $0.1 \%$ prevalence in the general population [43]. Sixteen of these 19 patients had a positive ID-Migraine score, which is not surprising considering the high incidence of nausea and photophobia occurring during cluster headache attacks, described first by Bahra et al. [44], and also corroborated by a Hungarian study [45] where the prevalence of nausea and photophobia during a cluster headache attacks were $43 \%$ and $68 \%$, respectively. As shown in the Results section, eliminating the cluster headache patients resulted in a significant rise in the specificity, from $42 \%$ to $52 \%$, while, interestingly, the NPV did not change much. Given the fact that the spontaneous occurrence of cluster headache in a similar sample from the general population would not be expected to be more than 1 patient, it may be safe to suggest that the specificity of the Hungarian version may be noticeably higher in representative samples.

Another issue affecting the specificity and NPV of the patients may have been a diagnostic error in chronic TTH patients. As outlined in the Results, 13 of the 19 chronic TTH patients with a positive ID-Migraine score could be considered migraineurs based on the characteristics of their headaches. As follow up data were not available for most TTH patients, this suggestion could 
neither be proved or disproved: however it is plausible that patients having chronic TTH and episodic migraines may not have had the latter diagnosis during their clinical visit. Our clinical experience with patients (not taking part in this study) who are followed up with a headache diary is that at least $20 \%$ of the patients who describe only migraine during the first visit would eventually be fund to have tension type headaches (TTH) as well, and a smaller percentage of patients originally diagnosed as 'pure' chronic TTH would also have attacks that fulfil the criteria of migraine. (This experience is not adequately reflected in the study as the number of patients included in the test-retest reliability part is still quite small.) These observations primarily affect the positive and negative predictive value and may increase the false positive test ratio, thus reducing the specificity of the questionnaire, and increasing its sensitivity and classification error.

A major limitation of our study is that our patients were recruited from patients visiting specialist headache services, so the sample is not representative of the general population. In particular, patients with episodic TTH were hugely underrepresented, and cluster headache patients were overrepresented. It is to be expected that, regardless of the diagnosis, patients with more severe head pain and accompanying symptoms would be overrepresented, and this may result in better quality indicators.

A further major limitation, that concerns the applicability of the ID-Migraine in the Hungarian population, is represented by the low specificity and low negative predictive value observed in our sample, the reasons of which are discussed above. The fact that test-retest reliability could only be tested in $10.5 \%$ of the patients represents a further limitation, although this percentage is actually slightly higher, than in the Portuguese validation study [19]. Finally, the fact that a minority of patients filled in the questionnaire after the medical visit is also a limitation, because the questions asked by the neurologist may have reinforced the patients' memories of the characteristics of headache that are the items in the IDMigraine questionnaire.

\section{Conclusion}

Our validation study proved that the Hungarian version of the ID-Migraine Questionnaire is a reliable tool to screen migraine patients in Hungary, with a high sensitivity and positive predictive value. However, mainly because of the low specificity observed in the current study, using ID-Migraine as a standalone diagnostic tool in Hungarian patients is currently not feasible. Further testing of the instrument is required, preferably in a sample from the general population.

\begin{abstract}
Abbreviations
$\mathrm{Cl}$ : Confidence interval; ICHD3- $\beta$ : International Classification of Headache Disorders; $\mathrm{MOH}$ : Medication overuse headache; NI: No information; NPV: Negative predictive value; PPV: Positive predictive value; ROC: Receiver operating curve; SUNCT: Short-lasting unilateral neuralgiform headache with conjunctival injection and tearing
\end{abstract}

\section{Funding \\ No funding.}

\section{Availability of data and materials}

The datasets generated and/or analysed during the current study are not publicly available due to the privacy of the data, but are available from the corresponding author on reasonable request.

\section{Authors' contributions}

CE, EC, GB, TG, MM and MT made substantial contributions to conception and design, acquisition of data, analysis and interpretation of data, and drafted the manuscript. CE, DB and GJ revised it critically for important intellectual content, gived final approval of the version to be published, and agreed to be accountable for all aspects of the work in ensuring that questions related to the accuracy or integrity of any part of the work are appropriately investigated and resolved. All authors read and approved the final manuscript.

Ethics approval and consent to participate

The study protocol had been approved by the ethics committee of Semmelweis University.

Consent for publication

Each participant gave informed consent to processing their results.

\section{Competing interests}

The authors declare that they have no competing interests.

\section{Publisher's Note}

Springer Nature remains neutral with regard to jurisdictional claims in published maps and institutional affiliations.

\section{Author details}

${ }^{1}$ Szentágothai János Doctoral School of Neurosciences, Semmelweis University, Üllői u. 26, Budapest 1085, Hungary. ${ }^{2}$ Department of Neurology, Vaszary Kolos Hospital, Petőfi Sándor u. 26-28, Esztergom 2500, Hungary. ${ }^{3}$ Department of Neurology, Semmelweis University, Balassa u. 6, Budapest 1083, Hungary. ${ }^{4}$ SE-NAP2 Genetic Brain Imaging Migraine Research Group, Semmelweis University, Nagyvárad tér 4, Budapest 1089, Hungary.

${ }^{5}$ Department of Pharmacodynamics, Faculty of Pharmacy, Semmelweis

University, Nagyvárad tér 4, Budapest 1089, Hungary.

Received: 29 July 2018 Accepted: 25 October 2018

Published online: 12 November 2018

\section{References}

1. Stovner L, Hagen K, Jensen R, Katsarava Z, Lipton R, Scher A et al (2007) The global burden of headache: a documentation of headache prevalence and disability worldwide. Cephalalgia 27(3):193-210

2. Diamond S, Bigal ME, Silberstein S, Loder E, Reed M, Lipton RB (2007) Patterns of diagnosis and acute and preventive treatment for migraine in the United States: results from the American migraine prevalence and prevention study. Headache 47(3):355-363

3. Osterhaus JT, Townsend RJ, Gandek B, Ware JE, Jr. Measuring the functional status and well-being of patients with migraine headache. Headache 1994; 34(6):337-343

4. Bank J, Marton S (2000) Hungarian migraine epidemiology. Headache 40(2): 164-169

5. Steiner TJ, Stovner LJ, Vos T (2016) GBD 2015: migraine is the third cause of disability in under 50s. J Headache Pain. 17(1):104

6. Linde M, Dahlof C (2004) Attitudes and burden of disease among selfconsidered migraineurs--a nation-wide population-based survey in Sweden. Cephalalgia 24(6):455-465 
7. Hu XH, Markson LE, Lipton RB, Stewart WF, Berger ML. Burden of migraine in the United States: disability and economic costs. Arch Intern Med1999; 159(8):813-8

8. Lipton RB, Stewart WF, Diamond S, Diamond ML, Reed M (2001) Prevalence and burden of migraine in the United States: data from the American migraine study II. Headache 41(7):646-657

9. Lipton RB, Diamond S, Reed M, Diamond ML, Stewart WF (2001) Migraine diagnosis and treatment: results from the American migraine study II. Headache 41:638-645

10. Lipton RB, Scher Al, Kolodner K, Liberman J, Steiner TJ, Stewart WF (2002) Migraine in the United States: epidemiology and patterns of health care use. Neurology 58(6):885-894

11. Latinovic R, Gulliford M, Ridsdale $L$ (2006) Headache and migraine in primary care: consultation, prescription, and referral rates in a large population. J Neurol Neurosurg Psychiatry 77(3):385-387

12. Steiner TJ, Scher Al, Stewart WF, Kolodner K, Liberman J, Lipton RB (2003) The prevalence and disability burden of adult migraine in England and their relationships to age, gender and ethnicity. Cephalalgia 23(7):519-527

13. Lipton RB, Stewart WF, Simon D (1998) Medical consultation for migraine: results from the American migraine study. Headache 38(2):87-96

14. Kobak KA, Katzelnick DJ, Sands G, King M, Greist JJ, Dominski M (2005) Prevalence and burden of illness of migraine in managed care patients. J Manag Care Pharm 11(2):124-136

15. Smetana GW (2000) The diagnostic value of historical features in primary headache syndromes: a comprehensive review. Arch Intern Med 160(18): 2729-2737

16. Khu JV, Siow HC, Ho KH (2008) Headache diagnosis, management and morbidity in the Singapore primary care setting: findings from a general practice survey. Singap Med J 49(10):774-779

17. Lipton RB, Dodick D, Sadovsky R, Kolodner K, Endicott J, Hettiarachchi J et al (2003) A self-administered screener for migraine in primary care: the ID migraine validation study. Neurology 61(3):375-382

18. Brighina F, Salemi G, Fierro B, Gasparro A, Balletta A, Aloisio A et al (2007) A validation study of an Italian version of the "ID migraine". Headache 47(6): 905-908

19. Gil-Gouveia R, Martins I (2010) Validation of the Portuguese version of IDmigraine. Headache 50(3):396-402

20. Karli N, Ertas M, Baykan B, Uzunkaya O, Saip S, Zarifoglu M et al (2007) The validation of ID migraine screener in neurology outpatient clinics in Turkey. J Headache Pain. 8(4):217-223

21. Di Piero V, Altieri M, Conserva G, Petolicchio B, Di Clemente L, Hettiarachchi $J$ (2007) The effects of a sensitisation campaign on unrecognised migraine: the Casilino study. J Headache Pain 8(4):205-208

22. Cousins G, Hijazze S, Van de Laar FA, Fahey T (2011) Diagnostic accuracy of the ID migraine: a systematic review and meta-analysis. Headache 51(7): 1140-1148

23. Mostardini C, d'Agostino VC, Dugoni DE, Cerbo R (2009) A possible role of ID-migraine in the emergency department: study of an emergency department out-patient population. Cephalalgia 29(12): $1326-1330$

24. Kim ST, Kim CY (2006) Use of the ID migraine questionnaire for migraine in TMJ and orofacial pain clinic. Headache 46(2):253-258

25. Ertas M, Baykan B, Tuncel D, Gokce M, Gokcay F, Sirin H et al (2009) A comparative ID migraine screener study in ophthalmology, ENT and neurology out-patient clinics. Cephalalgia 29(1):68-75

26. Zarifoglu M, Karli N, Taskapilioglu O (2008) Can ID migraine be used as a screening test for adolescent migraine? Cephalalgia 28(1):65-71

27. Juhasz G, Csepany E, Magyar M, Edes AE, Eszlari N, Hullam G et al (2017) Variants in the CNR1 gene predispose to headache with nausea in the presence of life stress. Genes Brain Behav 16(3):384-393

28. Anthoine E, Moret L, Regnault A, Sebille V, Hardouin JB (2014) Sample size used to validate a scale: a review of publications on newly-developed patient reported outcomes measures. Health Qual Life Outcomes 12:176

29. Csepany E, Bozsik G, Kellermann I, Hajnal B, Scheidl E, Palasti A, Toth M et al (2014) Examining the diagnostic accuracy of a new migraine screener. Ideggyogy Sz 67(7-8):258-268

30. Landis JR, Koch GG (1977) The measurement of observer agreement for categorical data. Biometrics 33(1):159-174

31. Diener HC, Dowson A, Whicker S, Bacon T (2008) Development and validation of a pharmacy migraine questionnaire to assess suitability for treatment with a triptan. J Headache Pain. 9(6):359-365
32. ligaya M, Sakai F, Kolodner KB, Lipton RB, Stewart WF (2003) Reliability and validity of the Japanese migraine disability assessment (MIDAS) questionnaire. Headache 43(4):343-352

33. Zandifar A, Asgari F, Haghdoost F, Masjedi SS, Manouchehri N, Banihashemi $M$ et al (2014) Reliability and validity of the migraine disability assessment scale among migraine and tension type headache in Iranian patients. Biomed Res Int 2014:978064

34. Manhalter N, Bozsik G, Palasti A, Csepany E, Ertsey C (2012) The validation of a new comprehensive headache-specific quality of life questionnaire. Cephalalgia 32(9):668-682

35. Lipton RB, Buse DC, Saiers J, Fanning KM, Serrano D, Reed ML (2013) Frequency and burden of headache-related nausea: results from the American migraine prevalence and prevention (AMPP) study. Headache 53(1):93-103

36. Pryse-Phillips W, Aube M, Bailey P, Becker WJ, Bellavance A, Gawel M et al (2006) A clinical study of migraine evolution. Headache 46(10):1480-1486

37. Holroyd KA, Drew JB, Cottrell CK, Romanek KM, Heh V (2007) Impaired functioning and quality of life in severe migraine: the role of catastrophizing and associated symptoms. Cephalalgia 27(10):1156-1165

38. Reed ML, Fanning KM, Serrano D, Buse DC, Lipton RB (2015) Persistent frequent nausea is associated with progression to chronic migraine: AMPP study results. Headache 55(1):76-87

39. Kelman $L$, Tanis D (2006) The relationship between migraine pain and other associated symptoms. Cephalalgia 26(5):548-553

40. Mattos A, Souza JA, Moreira PFF, Jurno ME, Velarde LGC (2017) ID-Migraine questionnaire and accurate diagnosis of migraine. Arq Neuropsiquiatr 75(7): 446-450

41. Wang X, San YZ, Sun JM, Zhou HB, Li X, Zhang ZM et al (2015) Validation of the Chinese version of ID-migraine in medical students and systematic review with meta-analysis concerning its diagnostic accuracy. J Oral Facial Pain Headache 29(3):265-278

42. Jurno M, Moreira Filho P, Ferreira A, Mattos AC, Souza J, Rezende D. Utility of ID-migraine as a screening tool in the specialty care (P04.253). Neurology 2012;78(1 Supplement):P04.253

43. Manzoni GC, Stovner LJ (2010) Epidemiology of headache. Handb Clin Neurol 97:3-22

44. Bahra A, May A, Goadsby PJ (2002) Cluster headache: a prospective clinical study with diagnostic implications. Neurology 58(3):354-361

45. Ertsey C, Vesza Z, Bangó M, Varga T, Nagyidei D, Manhalter N et al (2012) A prospective study evaluating the clinical characteristics of cluster headache. Ideggyogy Sz. 65(9-10):307-314

\section{Ready to submit your research? Choose BMC and benefit from:}

- fast, convenient online submission

- thorough peer review by experienced researchers in your field

- rapid publication on acceptance

- support for research data, including large and complex data types

- gold Open Access which fosters wider collaboration and increased citations

- maximum visibility for your research: over $100 \mathrm{M}$ website views per year

At $\mathrm{BMC}$, research is always in progress.

Learn more biomedcentral.com/submission 